\title{
Vergleichende Untersuchungen über den oralen und intravenösen Tolbutamidtest*
}

\author{
II. Bestimmung von Glucose, Serum-Insulin und Serum-Tolbutamid bei normalen, jungen Probanden
}

\author{
P. Berohtold, V. BÜBer, V. Meier, J.-P. Felber und G. Keiser
} Medizinische Abteilung, Bürgerspital, Zug/Schweiz (Chefarzt: Priv.-Doz. Dr. G. Keiser) und Département de Biochimie
Clinique, Clinique Médical Universitaire, Lausanne/Suisse (Directeur: Prof. A. Vannotti)

Eingegangen am 4. Juni 1970, angenommen am 10. Dezember 1970

Comparative investigations concerning the oral and $i . v$. tolbutamide tests.II. Determination of glucose, serum-insulin and serum tolbutamide in normal young subjects

Summary. In 12 normal subjects the oTT and ivTT were studied by simultaneous determination of blood glucose, serum insulin (IRI) and serum tolbutamide. From the results it is possible, to deduce two different mechanisms for the action of the orally administred tolbut. amide. They are dependent of the absorption rate of the drug. When tolbutamide is absorbed quickly, insulin secretion follows. It is responsible for the fall of blood sugar. When the tolbutamide absorption is slow, insulin secretion is small, but the blood sugar level is still falling. The three following hypotheses are proposed: either the insulin which appears in the pancreatic vein cannot be measured at the periphery and acts mostly in the liver, or the low concentration of tolbutamide in the serum causes only a slight secretion of insulin, but an important inhibition of the secretion of glucagon which is responsible for the observed hypoglycaemia. Extrapancreatic action of sulphonylurea compounds, particularly on muscle tissue, may too play an important role to explain the results.

Etudes comparatives du test au tolbutamide oral et intraveineux. II. Dosage du glucose, de l'insuline et du tolbutamide dans le sérum, chez des sujets jeunes normaux

Résumé. Chez 12 sujets normaux l'oTT et l'ivTT ont été étudiés en déterminant simultanément la glycémie, l'insuline sérique (IRI) et le tolbutamide du sérum. D'après les résultats il est possible de déduire deux mécanismes d'action différents du tolbutamide administré oralement. Ils dépendent de la vitesse d'absorption de la drogue. Quand le tolbutamide est absorbé rapidement, il se produit une sécretion d'insuline quiest responsable de la chute de la glycémie. Quand l'absorption du tolbutamide est lente, la sécrétion d'insuline est faible, mais le taux de la glycémie diminue quand même. Les trois hypothèses suivantes sont proposées: soit l'insuline qui apparaît dans la veine pancréatique ne peut pas être mesurée à la périphérie et agit surtout dans le foie, soit la faible concentration de tolbutamide dans le sérum ne provoque qu'une légère sécrétion d'insuline, mais une inhibition importante de la sécrétion de glucagon, qui est responsable de l'hypoglycémie observée. L'action extra-pancréatique des sulfonylurées, en particulier sur le tissu musculaire, peut également jouer un rôle important dans l'explication de ces résultats.

Zusammenfassung. Bei 12 normalen Probanden wurde mittels der Bestimmung von Glucose, Serum-Insulin und Serum-Tolbutamid der orale und der intravenöse Tolbutamidtest vergleichend untersucht. Aus den Resultaten ergeben sich zwei verschiedene Wirkungsmechanismen, die von der Tolbutamid-Resorptionsrate abhängig sind. Wird Tolbutamid rasch resorbiert, so erfolgt eine Insulinsekretion, die für den Blutzuckerabfall verantwortlich ist. Geht die Tolbutamidresorption langsam vor sich, so ist die Insulinsekretion gering, aber der Blutzucker fällt trotzdem ab. Zur Erklärung werden drei Hypothesen diskutiert: Entweder ist das Insulin, das in die Pankreasven $\theta$ sezerniert wird, peripher nicht meßbar und entfaltet seine Wirkung allein an der Leber, oder die niedere Serum-Tolbutamidkonzentration verursacht nur eine unwesentliche Insulinsekretion, dafür eine Hemmung der Glucagonsekretion, die ihrerseits für die Hypoglykämie verantwortlich ist. Auch extrapancreatische Wirkungen der Sulfonylharnstoffe, besonders die auf das Muskelgewebe, werden diskutiert.

Key-words: Intravenous tolbutamide test, oral tolbutamide test, glucose, serum-insulin, serum tolbuta. mide, tolbutamide action.
Ungenügende Resultate aus einer klinischen Vergleichsuntersuchung [4] von oralem Tolbutamidtest (oTT) und intravenösem Tolbutamidtest (ivTT) gaben Anlaß, den oTT und den ivTT durch Bestimmung von Glucose, Serum-immuno-reaktivem-Insulin und SerumTolbutamid in kurzen Abständen zu untersuchen.

Auf i.v. Verabreichung von Tolbutamid erfolgt eine rasch einsetzende Erniedrigung des Blutzuckers die mit einer Insulinausschüttung des Pankreas korreliert

* Teile dieser Arbeit wurden am 7. Kongress der internationalen Diabetes Federation (IDF) in Buenos Aires, 1970 vorgetragen. ist $[17,22,24,25,29,30,32,33,40,43]$. Vecchio et al. [41] bestimmten bei 5 Probanden Glucose und SerumTolbutamid während dem oTT und ivTT. 10 min nach oraler Tolbutamidgabe betrug die Serum-Tolbutamidkonzentration $10 \mathrm{mg} / 100 \mathrm{ml}$, nach $20 \mathrm{~min} 20 \mathrm{mg} / 100$ $\mathrm{ml}$ und nach $40 \mathrm{~min} 22 \mathrm{mg} / 100 \mathrm{ml}$. Beim ivTT fiel die Serum-Tolbutamidkonzentration von einem $10 \mathrm{~min}$ Wert von $18 \mathrm{mg} / 100 \mathrm{ml}$ auf $14 \mathrm{mg} / 100 \mathrm{ml}$ nach $50 \mathrm{~min}$. Somit war bei oraler Gabe von $2 \mathrm{~g}$ Tolbutamid der Serumspiegel nach $30 \mathrm{~min}$ höher als nach $1 \mathrm{~g}$ Tolbutamid i. v.. Insulinbestimmungen beim oTT wurden von Gundersen [13] durchgeführt. Er bestimmte die ILA 
nach Vallance-Owen nüchtern und 30 bis 40 min nach oraler Tolbutamidgabe. Der ILA-Anstieg war gering, während der Blutzuckerabfall bei normalen Probanden $41 \%$ des Nüchternwertes betrug.

Die Resultate der vorliegenden Untersuchung weisen darauf hin, daß bei oraler Tolbutamidapplikation verschiedene Mechanismen eine Rolle spielen, die möglicherweise die schlechte Utbereinstimmung von oTT und ivTT in der klinischen Studie [4] erklären können.

\section{Methodik}

Bei 12 Probanden (6 Frauen und 6 Männer) mit einem mittleren Alter von 26 Jahren (Bereich 19-37 Jahre) und einem mittleren Gewicht von $60 \mathrm{~kg}$ (Bereich $48-73 \mathrm{~kg}$ ) wurden ein oraler Glucosetoleranztest, (oGTT) mit $50 \mathrm{~g}$ Glucose und der ivTT sowie der oTT wie folgt durchgeführt:

Beim ivTT wurde nach 10 bis 12-stündigem Fasten (Haas [14]) am liegenden Probanden ein kurzer, mit Mandrin verschließbarer Verweillkatheter (Braunüle) in eine Kubitalvene eingelegt und während der Dauer des iv'TT belassen. Am anderen Arm wurde $1 \mathrm{~g}$ Tolbutamid in $20 \mathrm{ml}$ während 2 min i.v. gespritzt. Blutentnahme nüchtern, 2, 5, 10, 20, 30, 60 und $120 \mathrm{~min}$ nach Tolbutamidinjektion.

Beim $o T T$ wurde nach gleicher Vorbereitung, wie für den ivTT $1.5 \mathrm{~g}$ Tolbutamid und $3 \mathrm{~g}$ NatriumBicarbonat mit ca. $100 \mathrm{ml}$ Wasser oral verabreicht. Blutentnahmen nüchtern, 2, 5, 10, 15, 20, 30, 40, 50, $60,80,120$ und 140 min nach Tolbutamid und Bicarbonat-Aufnahme. Beide Teste wurden mit einem reichhaltigen Frühstück abgeschlossen. Im Venenblut wurden folgende Bestimmungen durchgeführt: Glucose mit der Glucose-Oxydase Methode [35] im Autoanalyzer (Technicon), IRI nach Hales und Randle [15], Tolbutamid ${ }^{1}$ nach Spingler [39].

Das Blut für die Insulin- und Tolbutamidbestimmung wurde 30 min bei Zimmertemperatur stehen gelassen, dann zentrifugiert und das Serum bei $-20^{\circ} \mathrm{C}$ gefroren. Alle Seren wurdem im gleichen Analysengang verarbeitet. Glucose wurde sogleich nach der Blutentnahme bestimmt.

Bei 2 Kontrollprobanden, einer 28-jährigen Frau und einem 25-jährigen Mann, die identisch sind mit Proband Nr. 8 und 12, wurde die gleiche Versuchsanordnung wie beim ivTT und oTT gewählt, aber an Stelle von Tolbutamid wurden einerseits $20 \mathrm{ml} \mathrm{NaCl}$ i.v. gespritzt, andererseits $3 \mathrm{~g}$ Natrium-Bicarbonat ohne Tolbutamid oral mit circa $100 \mathrm{ml}$ Wasser verabreicht.

Bei allen Probanden wurden die Transaminasen SGOT und SGPT bestimmt.

1 Tolbutamid wurde von dipl. chem. P. Hajdú, Farbwerke Hoechst A.G. Frankfurt/Main, bestimmt.

\section{Resultate}

Die Transaminasen waren normal. SGOT Mittelwert $8 \mathrm{IE} \pm 1.93 \mathrm{SD}$, SGPT $6 \mathrm{IE} \pm 2.15 \mathrm{SD}$ (Normalwerte für SGOT und SGPT: bis $12 \mathrm{IE})$.

Der $o G T T$ (Abb. 1) ist bei allen Probanden auf eine Glucosetoleranzstörung unverdächtig. Der oGTT der Kontrollen ist wegen der Identität mit Proband Nr. 8 und 12 normal.

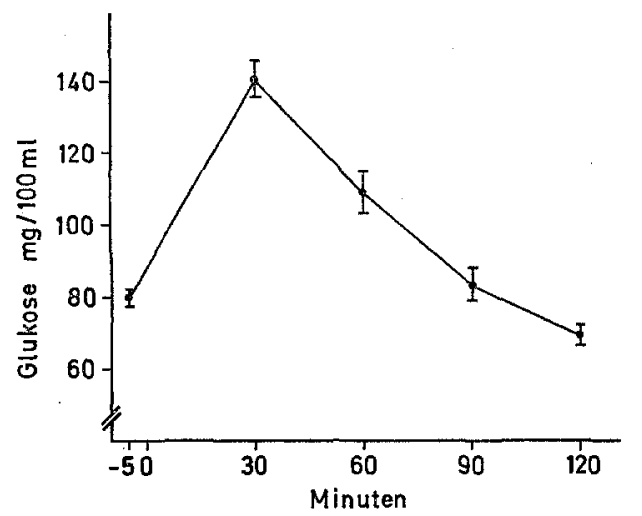

Abb. 1.50 g oraler Glucosetoleranztest von 12 jungen Probanden, Mittelwert $\pm \mathrm{SEM}$

Da sich Proband Nr. 5 während des oTT anders verhält, wird er gesondert betrachtet und im Folgenden wird vorerst nur über die restlichen 11 Probanden berichtet.

Beim $i v T T$ (Abb. 2) steigt nach Injektion von $1 \mathrm{~g}$ Tolbutamid die Serum-Tolbutamidkonzentration nach 2 min steil auf $21.5 \mathrm{mg} / 100 \mathrm{ml} \pm 3.65 \mathrm{SD}$. Danach sinkt der Tolbutamidspiegel allmählich auf $13 \mathrm{mg} / 100 \mathrm{ml} \pm$ 1,8 SD bei $120 \mathrm{~min}$. Mit dem Tolbutamidanstieg erfolgt ein praktisch simultaner Serum-Insulinanstieg von 9.3 mikro $\mathrm{E} / \mathrm{ml} \pm 2.1 \mathrm{SD}$ nüchtern auf $70.4 \mu \mathrm{E} / \mathrm{ml}$ 士46.1 SD. 60 min nach Tolbutamidinjektion ist der Seruminsulinspiegel wieder im Bereich des Nüchternwertes. Der Blutzucker sinkt 5 min nach Tolbutamidinjektion, also kurz nach dem Erreichen des IRIGipfels, ab. Nach Erreichen des tiefsten Wertes bei 30 min erfolgt der Wiederanstieg allmählich.

Berechnet man den Blutzuckerverlauf als prozentuale Abweichung rom Nüchternblutzucker (Ább. 3), so ergibt sich beim ivTT die bekannte Kurve, die nach Belser und Berger [3] für diese Altersgruppe normal ist.

Beim oTT (Abb. 2) ist die erste minimale Tolbutamidkonzentration von $0.06 \mathrm{mg} / 100 \mathrm{ml} \pm 0.18 \mathrm{SD}$ im Serum $10 \mathrm{~min}$ nach oraler Gabe von $1.5 \mathrm{~g}$ Tolbutamid und $3 \mathrm{~g}$ Natrium-Bicarbonat meßbar und steigt langsam weiter bis auf $15.97 \mathrm{mg} / 100 \mathrm{ml} \pm 4.0 \mathrm{SD}$ nach 140 min. Das IRI verbleibt 20 min im Bereich des Nüchternwertes, dann steigt es diskret auf ein Maximum von $16.5 \mu \mathrm{E} / \mathrm{ml} \pm 4.3 \mathrm{SD}$ nach $50 \mathrm{~min}$. Nach $60 \mathrm{~min}$ sinkt der Seruminsulinspiegel, erreicht aber den Nüchternwert bis zum Testende nicht. Der Blutzucker bleibt 20 min nach Tolbutamidgabe im Bereich des Nüchternwertes, fällt dann allmählich ab, wobei der Abfall, im 
Gegensatz zum ivTT, gleichzeitig mit dem Insulinanstieg einsetzt. Der Tiefpunkt der Kurve liegt nach $80 \mathrm{~min}$ auf $60.5 \mathrm{mg} / 100 \mathrm{ml} \pm 12 \mathrm{SD}$. Danach erfolgt ein allmählicher Wiederanstieg auf $67.4 \mathrm{mg} / 100 \mathrm{ml} \perp$ 6.9 SD bei $140 \mathrm{~min}$.

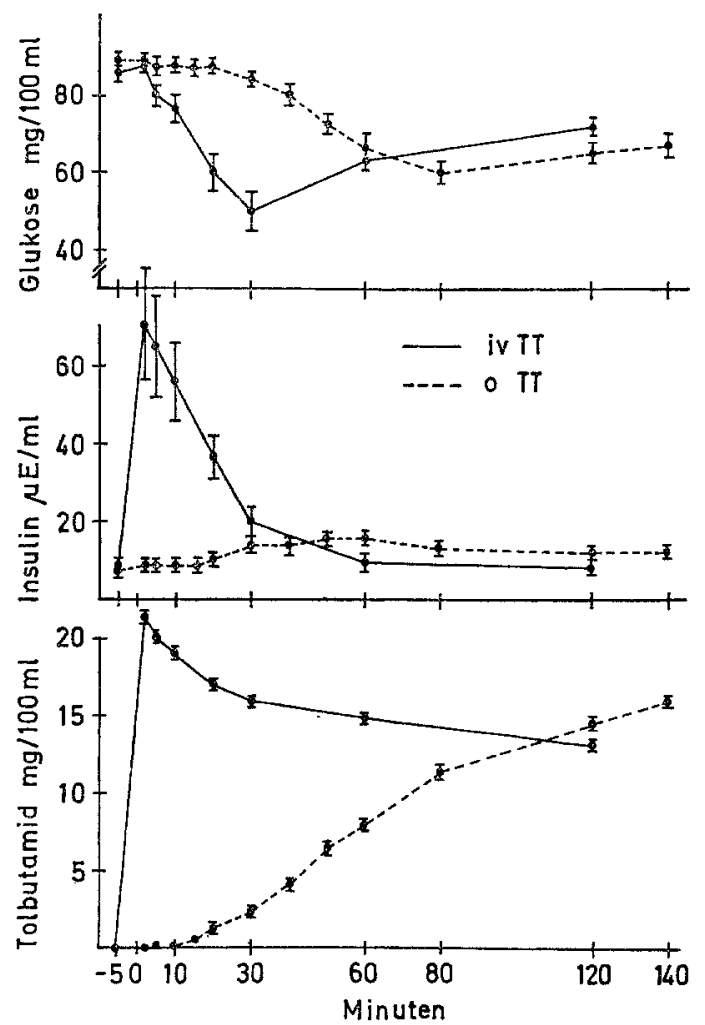

Abb. 2. Glucose, Serum-immuno-reaktives-Insulin und Serum-Tolbutamid während ivTT (ausgezogene Linie) und oTT (gestrichelte Linie) bei 11 Probanden. Mittelwerte \pm SEM

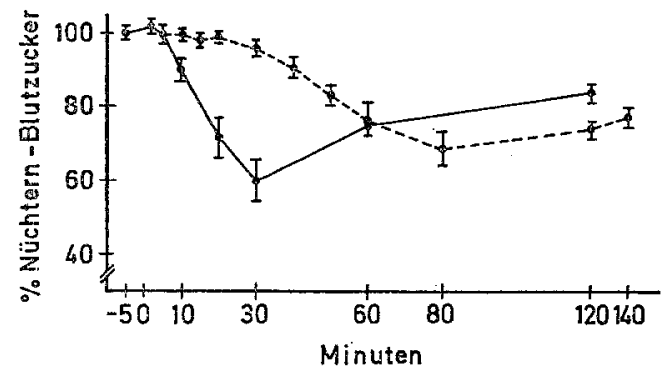

Abb. 3. Blutglucose in \% des Nüchtern-Blutzuckers während ivTT (ausgezogene Linie) und oTT (gestrichelte Linie) bei 11 Probanden. Mittelwert \pm SEM

Bei Proband Nr. 5 (Abb. 4 und 5) zeigen die drei Parameter im ivTT praktisch das gleiche Verhalten, wie die übrigen 11 Probanden. Die initiale Insulinausschüttung ist zweigipflig, was sonst nicht festgestellt werden konnte.

Beim oTT ist Tolbutamid im Serum erstmals nach $15 \mathrm{~min}$ mit $1.4 \mathrm{mg} / 100 \mathrm{ml}$ meßbar. Es erfolgt ein steiler Anstieg auf ein Plateau von $22.3 \mathrm{mg} / 100 \mathrm{ml}$ nach
$60 \mathrm{~min}$, das bis zu 120 min anhält und dann leicht abfällt. Gleichzeitig mit dem steilen Tolbutamidanstieg erfolgt der Anstieg des IRI auf $57 \mu \mathrm{E} / \mathrm{ml}$ nach 40 min. Nach raschem Abfall erreicht das IRI während der Testzeit den Nüchternwert nicht. Der tiefste Blut-

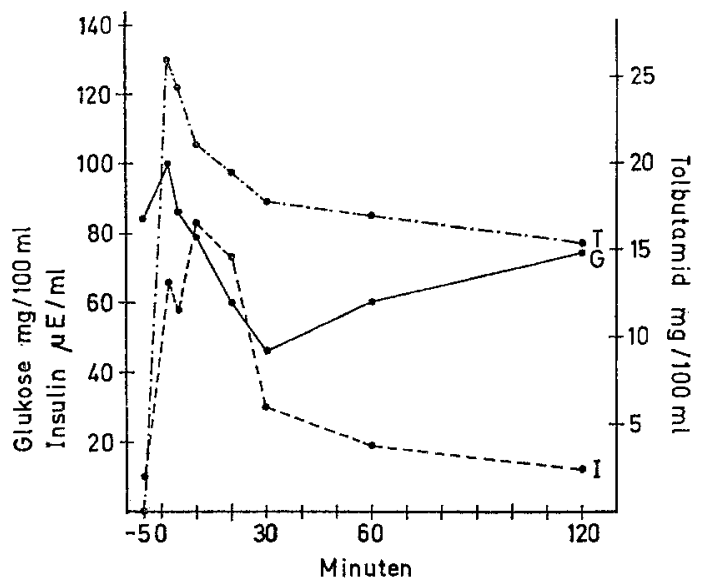

Abb. 4. Glucose (G), Serum-immuno-realttives- Insulin (I) und Serum-Tolbutamid (T) während des ivTT bei Proband 5

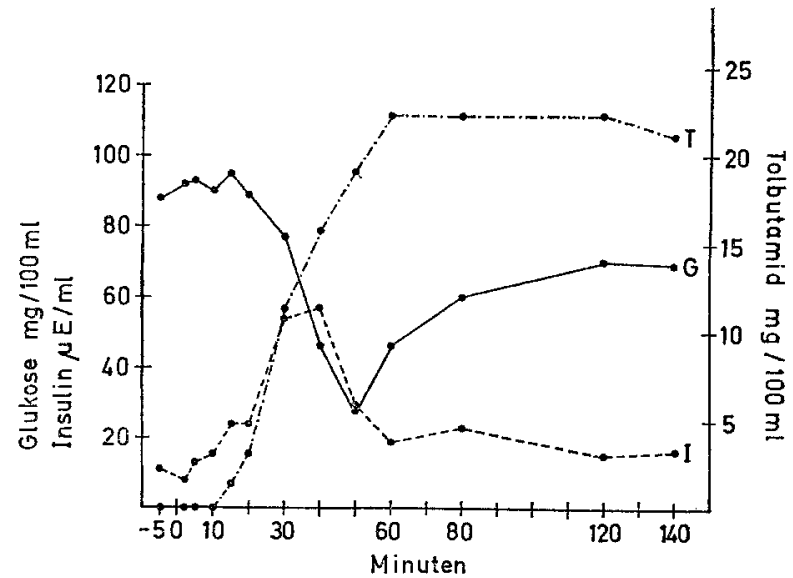

Abb. 5. Glucose (G), Serum-immuno-reaktives-Insulin (I) und Serum-Tolbutamid (T) während des oTT bei Proband 5. Differentes Verhalten gegenüber den anderen 11 Probanden (Abb. 2)

zuckerwert wird nach $50 \mathrm{~min}$ mit $28 \mathrm{mg} / 100 \mathrm{ml}$ gemessen.

Bei den Kontrollprobanden werden Serum-Insulin und Blutzucker durch Injektion von $20 \mathrm{ml} \mathrm{NaCl}$ oder orale Aufnahme von $3 \mathrm{~g}$ Natrium-Bicarbonat nicht verändert.

Die Werte sind in der gleichen zeitlichen Reihenfolge wie bei den anderen Tests angegeben und lauten (Mittelwert von 2 Probanden): $\mathrm{NaCl}$ i.v.: Blutglucose $86,92,90,88,92,92,93,88 \mathrm{mg} / 100 \mathrm{ml}$ IRI $11,8,9$, $10,9,10,7,8 \mathrm{mikroE} / \mathrm{ml}$.

Natrium-Bicarbonat oral: Blutzucker 90, 91, 90, $89,91,90,90,89,89,90,93,92,89 \mathrm{mg} / 100 \mathrm{ml}$.

IRI $7,7,10,7,8,8,7,9,8,7,9,7,7 \mu \mathrm{E} / \mathrm{ml}$. 


\section{Diskussion}

Nach intravenöser Verabreichung von Tolbutamid (Abb. 2 und 3), ist das Verhalten von Glucose [3], IRI [24] und Serumtolbutamid [41] mit den Befunden der Literatur übereinstimmend. Wird Tolbutamid jungen, normalen Probanden oral verabreicht, so lassen sich zwei verschiedene Reaktionsweisen beobachten. Sie sind von der Geschwindigkeit der Tolbutamidabsorption abhängig.

Die erste Reaktion, die bei Proband 5 (Abb. 5) abläuft, ist gekennzeichnet, durch rasche Tolbutamidresorption und raschem Anstieg des Serumtolbutamids, starkem Seruminsulinanstieg und sehr markanter Hypoglykämie. Der Gipfel des Insulinanstiegs liegt bei 30 bis $40 \mathrm{~min}$, was auch von Zeytinoglou und Gundersen [44] und von Otto et al. [28] nach oraler Verabreichung von verflüssigtem Glibenclamid festgestellt wurde.

Die zweite Reaktion, die bei 11 Probanden abläuft (Abb. 2), ist durch die langsame Tolbutamidresorption gekennzeichnet. Das Serumtolbutamid steigt, im Gegensatz zur ersten Reaktion, nur ganz allmählich und erreicht erst nach 40 bis 50 min wirksame Spiegel, die nach Sheldon et al. [36] zwischen 5.3 und $9.6 \mathrm{mg} / 100 \mathrm{ml}$ liegen.

Auf diesen verzögerten Tolbutamidanstieg erfolgt eine Erhöhung des Serum-Insulins, die aber nach 50 min nur das zweifache der Nüchternwerte erreicht, gegenüber einer zehnfachen Erhöhung des SerumInsulins nach intravenös verabreichtem Tolbutamid. Der Blutzucker sinkt aber trotzdem, proportional der Tolbutamidabsorptionsrate [8, 27], ab. Es handelt sich also um einen blutzuckersenkenden Tolbutamideffekt ohne wesentliche Erhöhung des Serum-Insulins.

Dies könnte mit einer isolierten Erhöhung des Serum Insulins in der Pankreasvene, die peripher nicht meßbar ist, erklärt werden, was von Pfeiffer et al. [31] am Hund nachgewiesen wurde. Eine zentrale Insulinwirkung auf die Leber mit Verminderung der Glucoseabgabe und Erhöhung der Glucoseaufnahme [16, 18, $19,38]$ wäre damit für die Hypoglykämie verantwortlich.

Samols et al. [34] konnten mit kleinen Dosen Tolbutamid eine Hemmung der Glucagonsekretion nachweisen. Ein Glucagonmangel führt zur Hypoglykämie $[12,23,26,37]$ und möglicherweise kommt der blutzuckersenkende Effekt von Tolbutamid über eine Glucagonhemmung zustande. Andere Autoren konnten allerdings diesen Mechanismus nicht nachweisen [1, $2,5,7]$.

Endlich müssen auch extrapankreatische Tolbutamidwirkungen zur Erklärung der Hypoglykämie ohne vorausgehenden Insulinanstieg in Betracht gezogen werden. Neben der Potenzierung exogenen oder endogenen Insulins durch Tolbutamid $[20,21]$ wird auch eine tolbutamidbedingte Verstärkung der Glucoseaufnahme in der quergestreiften Muskulatur beschrieben $[9,10]$. Der Metabolit Hydroxymethyltolbutamid stimuliert die Insulinsekretion gleich stark wie Tolbutamid, entwickelt aber nur 35\% der hypoglykämisierenden Wirkung von Tolbutamid [11]. Daraus wird für Tolbutamid neben der Beta-Zellenstimulation eine zusätzliche Wirkung postuliert.

Aus Abb. 2 geht hervor, daß der absolute Tolbutamidspiegel im Serum keinen Einfluß auf die Insulinsekretion hat, da $120 \mathrm{~min}$ nach oraler Verabreichung. von Tolbutamid beträchtliche Konzentrationen gemessen werden, Glucose und Insulin aber praktisch den Ausgangswerten entsprechen. Dieses Phänomen, das auch von Vinik et al. [42] beobachtet wurde, kann dadurch erklärt werden, daß die tolbutamidinduzierte Insulinsekretion vom Glucosespiegel abhängig ist und durch eine Hypoglykämie gehemmt wird [6].

Die Resultate weisen darauf hin, daß bei oraler Applikation von Sulfonylharnstoffen, je nach Resorptionsgeschwindigkeit verschiedene Mechanismen zur Auswirkung kommen. Eine hohe Resorptionsrate führt zur Insulinsekretion die peripher meßbar ist, eine niedrige Resorptionsrate führt entweder über eine zentrale Insulinwirkung auf die Leber, oder über eine Glucagonhemmung, oder über extrapankreatische Tolbutamidwirkung, möglicherweise auf die quergestreifte Muskulatur, zur Hypoglykämie.

Diese Arbeit wurde großzügig durch Hoechst Pharma AG., Zürich (Dr. F. Heitz) unterstützt. - Wir danken für vorzügliche technische Assistenz Frl. B. Tschudi und Frl. M. Ochsner, für zuverläßige Sekretariatsarbeit Fr. K. Springuel.

\section{Literatur}

1. Aguilar-Parada, E., Eisentraut, A.M., Unger, R.H.: Effect of HB 419 (Glibenclamide) induced hypoglycamia on pancreatic glucagon secretion. Horm. Metab. Res., Suppl. ad Vol. 1, 48-50 (1969).

2. - - Pancreatic glucagon secretion in normal and diabetic subjects. Amer. J. med. Soi. 257, 415-419 (1969).

3. Belser, F.G., Berger, W.: Der diagnostische Wert des intravenösen Tolbutamidtests. Schweiz. med. Wschr. 94, 1818-1824 (1964).

4. Berchtold, P., Meier, V., Büber, V., Felber, J.-P., Keiser, G.: Vergleichende Untersuchungen über den oralen und intravenösen Tolbutamidtest. I. Klinische Studie bei Patienten mittleren Alters. Diabetologia 7, $72-75$ (1971).

5. Buchanan, K.D., Vance, J.E., Dinstl, K., Williams, R.H.: Effect of blood glucose on glucagon secretion in anesthetized dogs. Diabetes 18, 11-18 (1969).

6. Cerasi, E., Chowers, I., Luft, R., Widström, A.: The significance of the blood glucose level for plasma insulin response to intravenously administred tolbutamide in healthy subjects. Diabetologia 5, 343-348 (1969).

7. Chesney, Th., Schofield, J.G.: Studies on the secretion of pancreatic glucagon. Diabetes 18, 627-632 (1969).

8. Creutzfeldt, W., Andreu-Kern, F., Discher, R.: Correlations of plasma levels of oral antidiabetic agents with blood sugar responses. Ann. N.Y. Acad. Sci. 82, 537$546(1959)$

9. Feldman, J.M., Lebovitz, H.E.: Appraisal of the extrapancreatic actions of sulfonylureas. Arch. intern Med. 123, 314-322 (1969). 
10. - - An insulin dependent effect of chronic tolbutamide administration on the skeletal muscle carbohydrate system. Diabetes 18, 84-95 (1969).

11. - - Biological activities of tolbutamide and its metabolites. Diabetes 18, 529-537 (1969).

12. Grollman, A., Mc Caleb, W.E., White, F.: Glucagon deficiency as a cause of hypoglycemia. Metabolism 13, $686-690$ (1964)

13. Gundersen, K.: Effect of tolbutamide on "free" and "complexed" serum insulin. Diabetes 15, 663-667 (1966).

14. Haas, H.G.: Der zeitliche Einfluß der Kohlehydrataufnahme auf den Blutzuckerabfall beim Tolbutamidtest. Schweiz. med. Wschr. 94, 317 -319 (1964).

15. Hales, C.N., Randle, P.J.: Immunoassay of insulin with insulin antibody precipitate. Biochem. J. 88, 137-146 (1963).

16. Jeffcoate, S.L., Moody, A.J.: The role of the liver in the disposal of orally administred ${ }^{14} \mathrm{C}$-Glucose in the normal rat. Diabetologia 5, 293-299 (1969).

17. Loubatière A.: Stimulations and inhibitors of insulin secretion, physiological and pharmacological interferences, synergisms and antagonisms. Acta diab. Iatina 5, suppl. 1, 220-255 (1968).

18. Madison, L.L.: The role of insulin in controlling carbohydrate metabolism in the liver: In: Leibel, B.S., Wrenshall, G.A. (ed.): On the nature and treatment of diabetes. Excerpta med. Found. p. 129-140 (1965).

19. - Role of insulin in the hepatic handling of glucose. Arch. intern. Med. 123, 284-292 (1969),

20. Madsen, J.: Extrapancreatic and intrapancreatic action of antidiabetic sulphonylureas, a review. Acta med. scand. suppl. 476, 109-122 (1967).

21. - Mode of action of blood glucose lowering sulfonamides. Other extrapancreatic effects. Acta diab. latina 6, suppl. 1, 415-428 (1969).

22. Maingay, D., de Ruyter, H. A., Touber, J.L., Croughs, R.J.M., Schopman, W., Lequin, R.M.: Rapid rise of insulin concentration in the plasma after intravenous administration of sodium tolbutamide. Lancet 1967 I, $361-362$.

23. Mc Quarrie, J., Bell, E.T., Zimmermann, B., Wright, W.S.: Deficieny of alpha cells of pancreas as possible etiologic factor in familial hypoglycemosis. Fed. Proc. $9,337(1950)$.

24. Melani, F.: Serum insulin concentration following intravenous administration of tolbutamide. Acta diab. latina 4, suppl. 1, 106-112 (1967).

25. - Lawecki, J., Bartelt, K.M., Pfeiffer, E.F.: Immunologisch meßbares Insulin (IMI) bei Stoffwechselgesunden, Fettsüchtigen und adipösen Diabetikern nach intravenöser Gabe von Glucose, Tolbutamid und Glucagon. Diabetologia 3, 422-426 (1967).

26. Mikami, S.I., Ono, K.: Glucagon deficiency induced by extirpation of alpha islets of the fowl pancreas. Endocrinology 71, 464-473 (1962).

27. Nelson, E., Knoechel, E.L., Hamlin, W.E., Wagner, J.G.: Influence of the absorption rate of tolbutamide on the rate of decline of blood sugar levels in normal humans. J. Pharmacol. Sci. 51, 509-514 (1962).

28. Otto, H., Halle, V., Thum, G.: Changes of serum insulin in healthy subjects after intravenous and peroral application of Glibenclamide (HB 419). Horm. Metab. Res. 1, suppl. 1, 81-84 (1969).

29. Perley, M., Kipnis, D.M.: Plasma insulin response to glucose and tolbutamide of normal weight and obese diabetic and nondiabetic subjects. Diabetes 15, 867874 (1966).

30. Pfeiffer, E.F., Pfeiffer, M., Ditschuneit, H., Ahn, Ch.-S.: Clinical and experimental studies of insulin secretion following tolbutamide and metahexamide administration. Ann. N.Y. Acad. Sci. 82, 479-495 (1959).

31. - Renold, A.E., Martin, D.B., Dagenais, Y., Meakin, J.W., Nelson, D.H., Shoemaker, G., Thorn, G.W.: Untersuchungen über die Rolle des Pankreas im Wir. kungsmechanismus blutzuckersenkender Sulfonylharnstoffe. In : Oberdisse K., Jahnke K. Diabetes mel. litus, p. 298-303. Stuttgart: Thieme (1959)

32. - Dynamics of insulin secretion in normal, obese and diabetic subjects following beta-cell stimulation. In: Butterfield W.H.J., van Westering W., Tolbutamide . . . after ten years. Excerpta med. Found. Int. con. gress series Nr. 149, p. 127. Amsterdam 1967.

33. Samols, E.: Immunochemical aspects of insulin. In: Leibel, B.S., Wrenshall G.A. (ed.): On the nature and treatment of diabetes. Excerpta med. Found. p. 227246 (1965).

34. - Tyler, J.M., Mialhe, P.: Suppression of pancreatic glucagon release by the hypoglycemic sulfonylureas. Lancet $1969 \mathrm{I}, 174-176$.

35. Schmidt, F.H.: Enzymatische Methoden zur Bestimmung von Blut- und Harnzucker unter Berücksichtigung von Vergleichsuntersuchungen mit klassischen Methoden. Internist 4, 554-559 (1963).

36. Sheldon, J., Anderson, J., Stoner, L.: Serum concentration and urinary excretion of oral sulfonylurea compound. Diabetes 14, 362-367 (1965).

37. Sokal, J.E. : Glucagon - An essential hormone. Amér. J. Med. 41, 331-341 (1966).

38. Söling, H.D.: Die Insulinwirkung auf das Lebergewebe. In : Pfeiffer, E.F.: Handbuch des Diabetes mellitus, Band I, p. 511. München: Lehmann 1969.

39. Spingler, H.: UỦber eine Möglichkeit zur colorimetrischen Bestimmung von N-(4-Methyl-Benzolsulfonyl)N'-Butyl-Harnstoff im Serum. Klin. Wschr. 35, 533$535(1957)$.

40. Vallance-Owen, J., Joplin, G.F., Fraser, R.: Tolbutamide control of diabetes mellitus. Lancet 1959 П, $584-586$.

41. Vecchio, T.J., Smith, D.L., Oster, H.L., Brill, R.: Oral sodium tolbutamide in the diagnosis of diabetes mellitus. Diabetes 13, 30-36 (1964).

42. Vinik, A.I., Jackson, W.P.U., Keller, P., Marine, N.: Metabolic studies in diabetics on tolbutamide. Com. parison of a single dose with a divided dose regimen. Diabetologia 4, 203-209 (1968).

43. Yalow, R.S., Black, H., Villazou, M., Berson, S.A.: Comparison of plasma insulin lovels following administration of tolbutamide and glucose. Diabetes $\mathbf{9}$, $356-362$ (1960).

44. Zeytinoglou, I., Gundersen, K. : A comparison of serum insulin levels in normal and untreated diabetics after oral glucose and tolbutamide. Diabetes 14, 470 (1965).

Dr. P. Berchtold

Washington Hospital Center

Clinical Pharmacology Unit

G. Hyman Research Building

110 Irving Street N.W.

Washington D.C. 20010/U.S.A. 